Physics

Physics Research Publications

\title{
Elastometric sensing using higher flexural eigenmodes of microcantilevers
}

\author{
M. Spletzer \\ A. Raman
}

R. Reifenberger 


\title{
Elastometric sensing using higher flexural eigenmodes of microcantilevers
}

\author{
Matthew Spletzer and Arvind Raman ${ }^{\text {a) }}$ \\ School of Mechanical Engineering and Birck Nanotechnology Center, Purdue University, West Lafayette, \\ Indiana 47907, USA
}

\begin{abstract}
Ron Reifenberger
Department of Physics and Birck Nanotechnology Center, Purdue University, West Lafayette, Indiana 47907, USA
\end{abstract}

(Received 7 June 2007; accepted 5 October 2007; published online 1 November 2007)

\begin{abstract}
We present a method to detect with high sensitivity the viscoelastic property changes of sensor coatings on microcantilever arrays due to radiation, analyte binding, or adsorption. The method uses higher order flexural eigenmodes to identify the location and magnitude of the nonuniform elasticity changes in the microcantilever coating. We demonstrate the method by monitoring the time evolution of resonance frequencies and $Q$ factors of different flexural eigenmodes of microcantilevers functionalized with a small drop of a photosensitive polymer as it is exposed to ultraviolet radiation. The method is particularly well suited for measuring quantitatively the time varying viscoelastic properties of thin films or biological materials attached to microcantilevers.

(C) 2007 American Institute of Physics. [DOI: 10.1063/1.2803215]
\end{abstract}

Microcantilever-based resonant sensors are increasingly used as a versatile platform for the detection of targeted analytes that can be either biological or molecular in nature. Broadly speaking, dynamic microcantilever sensors can be classified as gravimetric or elastometric. Gravimetric sensing schemes detect the presence of the added mass of the target analyte through the accompanying decrease in microcantilever resonance frequencies. On the other hand, elastometric sensing schemes detect changes in elasticity of the microcantilever coating and the associated shifts in resonance frequencies. While the performance of gravimetric microcantilever sensors has been well studied, ${ }^{1,2}$ elastometric sensors have been less studied by comparison, ${ }^{1,3}$ and offer new possibilities for detecting biological analytes and radiation or measuring changes in cross linking or bond scission of thin polymer coatings. 4

In this article, we develop the basic theory of quantitative elastometric sensing and present a method that significantly enhances the sensitivity of elastometric sensing using microcantilevers. Specifically, we show that when the viscoelasticity of a patch of sensor coating changes due to exposure to radiation or analytes, then the relative resonance frequency and $Q$ factor shifts of the multiple eigenmodes not only provide highly sensitive measurements of the evolving elastic modulus and viscosity of the patch but they can also be used to identify the location of the patch. Experiments performed on microcantilevers coated with a liquid polymer that hardens upon exposure to ultraviolet light are used to verify the theoretical predictions.

The physics of the underlying problem of elastometric sensing is conveniently captured by modeling the nonuniform microcantilever as a Bernoulli-Euler beam. We focus on a simple case where a uniform beam, shown in Fig. 1(a), with bending stiffness $E I$ and mass per unit length $m$, is perturbed nonuniformly by a coating of different elasticity. Specifically, the coated section of the microcantilever $a<x$ $<a+d$ now possesses a different bending stiffness $(\hat{E I})$,

${ }^{\text {a)} E l e c t r o n i c ~ m a i l: ~ r a m a n @ e c n . p u r d u e . e d u ~}$ mass per unit length $(\hat{m})$, and viscosity $\hat{\alpha}$, as seen in Fig. 1(b). During elastometric sensing only the bending stiffness and viscosity of the coated section change to $\tilde{E I}$ and $\widetilde{\alpha}$, respectively. This scenario is commonly observed in elastometric sensors and is representative of the experiments described later.

The resonance frequency of the $i$ th eigenmode of the nonuniformly coated microcantilever shown in Fig. 1(b) can be found using Rayleigh's quotient. ${ }^{6}$ It is assumed that the added mass or elasticity due to the coating in the section $a<x<a+d$ is small enough that the eigenmodes remain close to those of a uniform beam. When this nonuniform microcantilever is exposed to radiation or analytes which affect only the bending stiffness of the coated section, the resonance frequency of the $i$ th eigenmode $\omega_{i}^{2}$ can again be calculated, this time using the new bending stiffness $\tilde{E I}$. The relative shift in the square of the resonance frequency of the $i$ th eigenmode is then given by:

$$
\frac{\omega_{i}^{2}-\omega_{0, i}^{2}}{\omega_{0, i}^{2}}=\frac{(\tilde{\gamma}-\hat{\gamma}) \int_{a}^{a+d} \psi_{i, x x}^{2} d x}{1+\hat{\gamma} \int_{a}^{a+d} \psi_{i, x x}^{2} d x},
$$

where $\hat{\gamma}=(\hat{E I} / E I)-1$ and $\tilde{\gamma}=(\tilde{E I} / E I)-1$ represent the relative shifts in bending stiffness after coating application and coating exposure, respectively, $\omega_{0, i}$ and $\omega_{i}$ represent the $i$ th resonance frequency of the microcantilever before and after coating exposure, respectively, $\psi_{i}$ represents the $i$ th eigenmode of a uniform microcantilever, and $\psi_{i, x x}=d^{2}\left(\psi_{i}\right) / d x^{2}$. Assuming small frequency shifts and small changes in elasticity of the coated section, we have $\left(\omega_{i}^{2}-\omega_{0, i}^{2}\right) / \omega_{0, i}^{2} \approx 2\left(\omega_{i}\right.$ $\left.-\omega_{0, i}\right) / \omega_{0, i}$, and Eq. (1) can be Taylor expanded for $\hat{\gamma}, \tilde{\gamma}$ $\ll 1$. Expression (1) further simplifies if it is assumed that the initial sensor coating does not influence the elasticity of the section $a<x<a+d$, but rather only its mass. ${ }^{8}$ Then we can set $\hat{\gamma}=0$ in Eq. (1), yielding the following formula for the relative frequency shift of the $i$ th microcantilever eigenmode: 


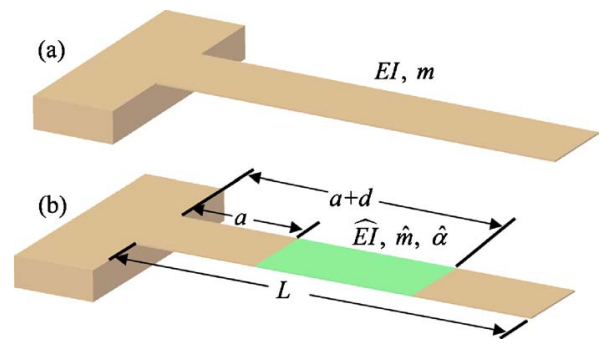

FIG. 1. (Color online) Schematic of a uniform microcantilever beam of length $L$ (a) prior to coating and (b) after coating. The dimensions $a$ and $a+d$ denote the coated section limits. The bending stiffness and viscosity of the coated section change to $\widetilde{E I}$ and $\widetilde{\alpha}$, respectively, after the coating is exposed to an analyte or radiation.

$$
\frac{\omega_{i}-\omega_{0, i}}{\omega_{0, i}}=\frac{\tilde{\gamma}}{2} \int_{a}^{a+d} \psi_{i, x x}^{2} d x .
$$

Next we derive a general expression for the shifts in $Q$ factor of different eigenmodes due to radiation induced changes in viscoelastic properties of the affected section. The mechanical power dissipated in the viscoelastic coating can be written in terms of an integral over the affected section, ${ }^{6}$ $P=A_{i}^{2} \omega_{0, i}^{2} \cos ^{2}\left(\omega_{0, i} t\right)(\hat{\alpha} \hat{E} I / 2) \int_{a}^{a+d} \psi_{i, x x}^{2} d x$, where $\hat{E I}$ and $\hat{\alpha}$ are the elastic modulus and viscosity, respectively, of the coated section of the beam before exposure, and $A_{i}$ is the oscillation amplitude of the $i$ th eigenmode. Equating the power dissipated to that of a damped simple harmonic oscillator, $P=A_{i}^{2}\left(\omega_{0, i}^{3} / Q\right) m_{\mathrm{eff}, i} \cos ^{2}\left(\omega_{0, i} t\right)$, yields the $Q$ factor of the $i$ th eigenmode due to the viscosity of the coating. A similar expression for the $Q$ factor after exposure can be obtained using $\widetilde{E I}$ and $\widetilde{\alpha}$. Assuming again that the coating has negligible bending stiffness prior to exposure $(\hat{\gamma}=0)$ and that $\tilde{\gamma}$ is small, the relative shift in the total quality factor of the $i$ th microcantilever eigenmode can be written as

$$
\begin{aligned}
\frac{\widetilde{Q}_{i}-\hat{Q}_{i}}{\hat{Q}_{i}}= & \frac{\widetilde{Q}_{i}}{2}\left[\hat{\alpha} \omega_{0, i}-\tilde{\alpha} \widetilde{\omega}_{i}(1+\tilde{\gamma}\right. \\
& \left.\left.+\tilde{\gamma} \int_{a}^{a+d} \psi_{i, x x}^{2} d x\right)\right] \int_{a}^{a+d} \psi_{i, x x}^{2} d x,
\end{aligned}
$$

where $\widetilde{Q}_{i}$ is the total quality factor of the $i$ th eigenmode after exposure and $\hat{Q}_{i}$ is the total quality factor of the $i$ th eigenmode prior to exposure. ${ }^{9}$ Both Eqs. (2) and (3) show that the relative shift in the resonance frequency and $Q$ factor of the $i$ th eigenmode depend on the relative change in viscoelasticity of the coated section as well as the location and extent of the coated section.

Let us now examine the theoretically predicted frequency and $Q$-factor shifts for different eigenmodes for a specific sensor configuration using Eqs. (2) and (3). For a fixed coated section size of $70 \mu \mathrm{m}$ and $\tilde{\gamma}=0.045$ based on experimental conditions ${ }^{10}$ described later, the resulting relative shifts in frequencies for the first three eigenmodes as a function of $a$ are plotted in Fig. 2(a). Figure 2(a) clearly shows that the eigenmode with greatest relative shift in resonance frequency depends sensitively on the location $a$ of the coated section. An eigenmode displays the greatest relative shift in resonance frequency when the coated section is located near an antinode of that eigenmode. In Fig. 2(b), the effect of the size of the coating section is considered. As Downloaded 03 Sep 2008 to 128.210.124.90. Redistribution subject
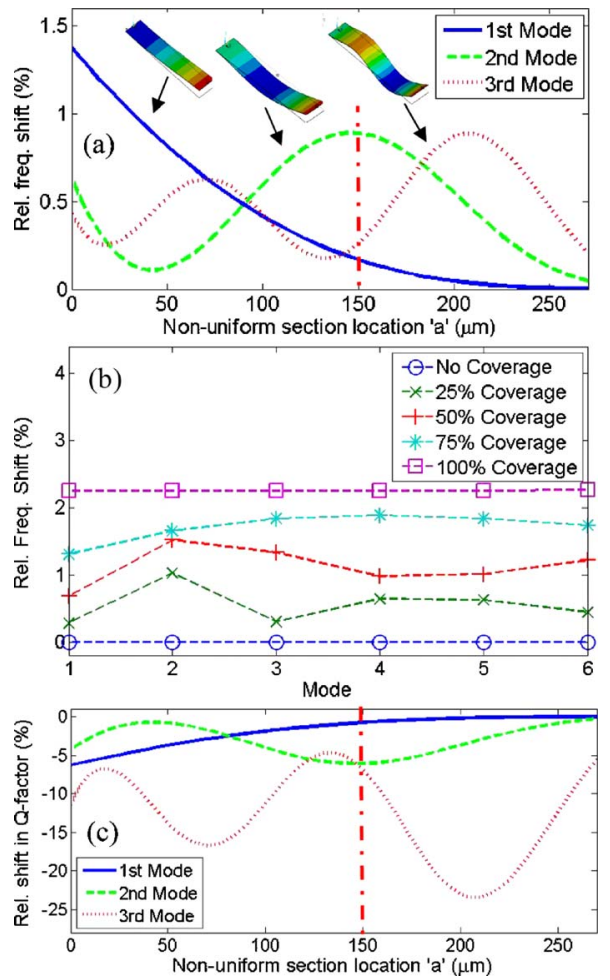

FIG. 2. (Color online) (a) Theoretically calculated relative resonant frequency shift vs coating location $a$ for the first three eigenmodes of a microcantilever beam. ${ }^{10}$ The values $d=70 \mu \mathrm{m}$ and $\tilde{\gamma}=0.045$ were selected based on experimental observations. (b) Theoretically calculated relative resonance frequency shift vs eigenmode for various percentages of nonuniform section coverage with $\tilde{\gamma}=0.045$. The covered portion is centered along the length of the microcantilever. (c) Theoretically calculated relative shift in $Q$ factor vs $a$ for the first three microcantilever eigenmodes. The properties of the microcantilever are identical to those used in (a), with the added conditions that $\hat{\alpha}=2.6 e-12 \mathrm{~s}$ and $\tilde{\alpha}=6 e-8 \mathrm{~s}$. The dashed lines in (a) and (c) represent the coating location in the experiments.

shown in Fig. 2(b) for the case of a uniformly coated microcantilever, the relative shifts in resonant frequencies are maximized and will be equal to $\tilde{\gamma} / 2$ for all eigenmodes measured. Moreover, nonuniform changes in elasticity or nonuniform coatings result in different relative frequency shifts in different eigenmodes while uniform changes or coatings result in identical relative frequency shifts for all eigenmodes. Consider relative $Q$-factor shifts using Eq. (3), where the properties of the microcantilever are identical to those used above, ${ }^{9,10}$ with the initial and final viscosities given by $\hat{\alpha}=2.6 e-12 \mathrm{~s}$ and $\tilde{\alpha}=6 e-8 \mathrm{~s},{ }^{11}$ respectively, and $\hat{\gamma}=0$ and $\tilde{\gamma}=0.045$, as shown in Fig. 2(c). The additional damping due to the increased viscosity of the nonuniform section causes a decrease in all of the quality factors calculated, with the nonuniform section location again dictating which eigenmode sees the greatest downward shift. Finally, from the measured resonant frequency and $Q$ factor shifts for several eigenmodes, Eqs. (2) and (3) can be inverted to determine the location $a$, size $d$, and relative change in bending stiffness and viscosity of the coated section.

In order to verify the theoretical predictions, experiments were performed for the elastometric sensing of incident radiation. Specifically, experiments were performed using microcantilevers ${ }^{10}$ which were coated with an UV-curing polymer $^{12}$ using the procedure outlined by Thundat and coworkers. ${ }^{3}$ The polymer formed a drop over a small portion of the microcantilever, as shown in Fig. 3(a), instead of a to AIP license or copyright; see http://apl.aip.org/apl/copyright.jsp 

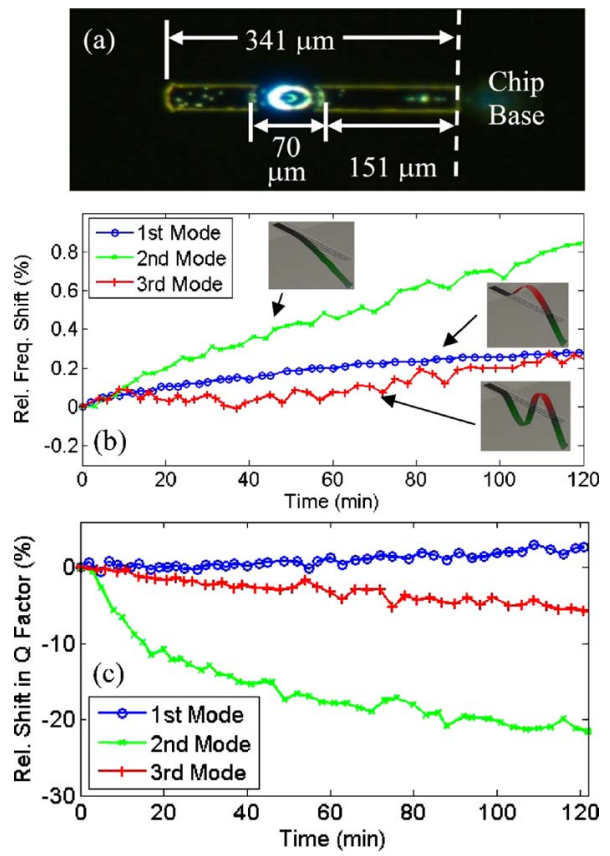

FIG. 3. (Color online) (a) Image of the microcantilever with the polymer drop applied. (b) Measured relative frequency shift vs UV radiation exposure time for the first three eigenmodes of the microcantilever in (a). The insets show the experimental mode shapes measured using a scanning laser Doppler interferometer of the microcanilever after the drop is fully cured. (c) Measured relative shift in $Q$ factors vs UV radiation exposure time for the first three modes of the microcantilever in (a).

uniform coating over the entire microcantilever.

The vibrations of the coated microcantilevers were analyzed using a Nanotec Electronica ${ }^{\mathrm{TM}}$ scanning probe microscopy system and a Polytec MSA400 scanning Doppler interferometer. The microcantilevers were exposed in situ to a UV-A lamp with a typical peak intensity of $470 \mu \mathrm{W} / \mathrm{cm}^{2}$ for approximately $2 \mathrm{~h}$. The resonance frequencies of the first three eigenmodes were measured before and after exposure, as well as at 3 min intervals during exposure. The eigenmodes of the microcantilevers before coating and after final curing were also measured using the MSA400 system and not found to change significantly.

Figure 3(b) shows the relative frequency shifts for all three peaks of the microcantilever as a function of exposure time along with the measured eigenmodes of the uncoated microcantilever. As expected, the curing of the polymer stiffened the center portion of the microcantilever, causing all of the frequencies to shift upward. The second eigenmode shows the highest relative frequency shift, while the first and third eigenmodes show nearly identical shifts. The vertical dashed line in Fig. 2(a) corresponds to the coating location in the experiment. Thus the theoretical model correctly predicts the ordering of the relative frequency shifts seen in the experimental data after $120 \mathrm{~min}$ of radiation exposure.

The relative shifts in the $Q$ factors of the microcantilever versus exposure time are shown in Fig. 3(c). As with the relative frequency shifts, the second eigenmode shows the greatest relative shift in $Q$ factor, followed by the third and first eigenmodes. The decrease in all measured $Q$ factors indicates that viscosity of the polymer increases significantly during the curing process. The vertical dashed line in Fig. 2(c) shows that the theoretical model again predicts the correct ordering of the relative $Q$ factor shifts seen in the experimental results after $120 \mathrm{~min}$ of exposure. perimental results after $120 \mathrm{~min}$ of exposure. cross section over the entire coated portion.
Downloaded 03 Sep 2008 to 128.210.124.90. Redistribution subject to AIP license or copyright; see http://apl.aip.org/apl/copyright.jsp
In the experiments discussed above, the observed relative frequency shifts correspond to a final increase of approximately $4 \%$ in the effective elastic modulus of the coated section of the beam, indicating the elastic modulus of the coating polymer has increased to approximately $50 \mathrm{kPa}^{13}$ Ultimately, the minimum detectable change in coating elasticity will depend on both the smallest resonance frequency shift that can be resolved experimentally and the percentage of the microcantilever that is coated. However since PPM frequency shifts are readily detectable for microcantilevers under ambient conditions, we anticipate that a similar change in coating elasticity will also be measurable.

In conclusion, we have demonstrated that measuring higher vibrational eigenmodes of microcantilevers can both detect and locate time-varying nonuniform changes in viscoelasticity. This method, in addition to allowing higher sensitivity elastometric detection of analytes located away from the free end of a microcantilever, is also capable of evaluating the uniformity of a microcantilever coating by simply measuring the pattern of relative frequency and $Q$ factor shifts of several eigenmodes. Knowledge of how resonance frequencies and $Q$ factors of several eigenmodes shift due to localized viscoelastic changes also allows the possibility of reliable detection of multiple analytes using a single microcantilever with multiple functionalized regions.

This work was partially supported by NASA Grant No. NCC 2-1363.

${ }^{1}$ B. Rogers, L. Manning, M. Jones, T. Sulchek, K. Murray, B. Beneschott, J. D. Adams, Z. Hu, T. Thundat, H. Cavazos, and S. C. Minne, Rev. Sci. Instrum. 74, 4899 (2003).

${ }^{2}$ F. M. Battiston, J.-P. Ramseyer, H. P. Lang, M. K. Baller, Ch. Gerber, J. K. Gimzewski, and H.-J. Güntherodt, Sens. Actuators B 77, 122 (2001); B. Ilic, D. Czaplewski, M. Zalalutdinov, and H. G. Craighead, J. Vac. Sci. Technol. B 19, 2825 (2001) L. B. Sharos, A. Raman, S. Crittenden, and R. Reifenberger, Appl. Phys. Lett. 84, 4638, (2004); S. Dohn, R. Sandberg, W. Svendsen, and A. Boisen, ibid. 86, 233501 (2005).

${ }^{3}$ A. Gupta, P. Nair, D. Akin, M. Ladisch, S. Broyles, M. Alam, and R. Bashir, Proc. Natl. Acad. Sci. U.S.A. 103, 13362 (2006); J. Tamayo, D. Ramos, J. Mertens, and M. Calleja, Appl. Phys. Lett. 89, 224104 (2006); T. Thundat, S. Sharp, W. Fisher, R. Warmack, and E. Wachter, ibid. 66, 1563 (1995).

${ }^{4}$ R. Benson, Nucl. Instrum. Methods Phys. Res. B 191, 752 (2002).

${ }^{5}$ G. Lubarsky, M. Davidson, and R. Bradley, Surf. Sci. 558, 135 (2004).

${ }^{6}$ L. Meirovitch, Principles and Techniques of Vibrations (Prentice Hall, Upper Saddle River, NJ, 1997), p. 237.

${ }^{7}$ The $i$ th eigenmode of a uniform microcantilever has been scaled such that $\int_{0}^{L} \psi_{i, x x}^{2} d x=1$.

${ }^{8}$ Experiments showed that applying the coating to the microcantilever caused the first three resonant frequencies to drop by $38 \%, 32 \%$, and $23 \%$, respectively. Thus, the added mass effect was the dominant effect observed, as is fairly common in practice where the coatings are very thin or in liquid state as in the experiments described.

${ }^{9}$ The total quality factor includes the effects of air and material damping in the microcantilever, which are assumed constant over the entire experiment in this case. The experimentally measured $\widetilde{Q}_{i}$, values were 55,13 , and 18 for the first, second, and third eigenmodes, respectively.

${ }^{10} \mathrm{CSC} 12$ tipless silicon $\left(E=112.4 \mathrm{GPa}, \rho=2329 \mathrm{~kg} / \mathrm{m}^{3}\right)$ microcantilevers from $\mu$ Masch, Inc. Microcantilever E (341 $\mu \mathrm{m}$ long, $35 \mu \mathrm{m}$ wide, and $1 \mu \mathrm{m}$ thick) was used in these experiments.

${ }^{11}$ The $\hat{\alpha}$, value corresponds to the manufacturer's specified dynamic viscosity of $0.3 \mathrm{Pas}$, and the value of $\widetilde{\alpha}$, was chosen based on experimental data and corresponds to a dynamic viscosity of $7080 \mathrm{Pas}$.

${ }^{12}$ Norland Optical Adhesive (NOA) 61, Norland Products, Inc., Cranbury, NJ. The elastic modulus of the polymer after complete curing is given as $1.03 \mathrm{GPa}$.

${ }^{13}$ The coated section was modeled as a composite beam with a constant 